\title{
The influence of cognition on self-management of type 2 diabetes in older people
}

This article was published in the following Dove Press journal:

Psychology Research and Behavior Management

21 January 2016

Number of times this article has been viewed

\author{
Ali Tomlin \\ Alan Sinclair \\ Institute of Diabetes for Older \\ People, University of Bedfordshire, \\ Luton, Bedfordshire, UK
}

Correspondence: Ali Tomlin Institute of Diabetes for Older People, University of Bedfordshire, Putteridge Bury Campus, Hitchin Road, Luton, Bedfordshire LU2 8LE, UK

Tel +44 I582489625

Fax +44 I582 743286

Email ali.tomlin@beds.ac.uk
Abstract: Diabetes is a growing public health issue, increasing in prevalence, eroding quality of life, and burdening health care systems. The complications of diabetes can be avoided or delayed by maintaining good glycemic control, which is achievable through self-management and, where necessary, medication. Older people with diabetes are at increased risk for cognitive impairment. This review aims to bring together current research that has investigated both cognition and diabetes self-management together. The Cumulative Index to Nursing and Allied Health (Cinahl), Excerpta Medica Database (Embase), Medical Literature Analysis and Retrieval System (Medline), and Psychological Information (PsychInfo) databases were searched. Studies were included if they featured older people with type 2 diabetes and had looked for associations between at least one distinct measure of cognition and at least one distinct measure of diabetes self-management. English language publications from the year 2000 were included. Cognitive measures of executive function, memory, and low scores on tests of global cognitive functioning showed significant correlations with multiple areas of diabetes self-management, including diabetes-specific numeracy ability, diabetes knowledge, insulin adjustment skills, ability to learn to perform insulin injections, worse adherence to medications, decreased frequency of self-care activities, missed appointments, decreased frequency of diabetes monitoring, and increased inaccuracies in reporting blood glucose monitoring. The nature of the subjects studied was quite variable in terms of their disease duration, previous medical histories, associated medical comorbidities, and educational level attained prior to being diagnosed with diabetes. The majority of studies were of an associational nature and not findings confirmed by repeat testing or by the effects of an intervention, neither were the majority of studies designed to give a view or conclusion on the clinical value or implications of the research. This only allows speculation of their importance. Most studies do not separate out the influence of aging itself in altering diabetes self-care behavior. We conclude that older people with type 2 diabetes are at increased risk for cognitive dysfunction. Changes in cognition may negatively affect diabetes self-management behaviors, influencing self-care outcomes. Age and depression may exacerbate any cognitive impairment.

Keywords: self-care, cognitive impairment, neuropsychological test, executive function

\section{Introduction}

Diabetes is a chronic and progressive condition and is increasingly prevalent, which can erode quality of life and place significant burden upon health care systems. The risk of developing complications of diabetes such as cardiovascular disease, neuropathy, retinopathy, and nephropathy can be lessened or delayed by good glycemic control. ${ }^{1}$ The condition is largely self-managed through diet and exercise and taking medications. 
Diabetes is associated with cognitive change, especially in those who are older and have had diabetes for longer. ${ }^{2}$ Specific areas of cognitive change seen in diabetes include verbal memory and psychomotor function. ${ }^{3}$ Cardiovascular disease and depressive symptomatology may influence some of the cognitive changes. ${ }^{4,5}$ Diabetes self-management tasks require multiple cognitive skills and processes, including memory, attention, planning, and calculation.

Although many researchers have looked at cognition in diabetes and self-management in diabetes, few have looked at the effects of changes in cognition upon self-management. There is little consensus on which areas of cognition and which self-management skills to measure and which assessment tools to use.

Research that highlighted associations between cognition and self-management could provide targets for interventions to improve self-management outcomes. This review therefore aims to investigate whether any change in cognition seen in type 2 diabetes in the older person affects the ability to self-manage the condition. We concentrate on studies that have each included adult participants with type 2 diabetes, at least one specific measure of cognitive function, and at least one specific measure of self-management. Cross-sectional study designs have been included, since the review is interested in associations between cognition and self-management. The review does not focus on interventions or succeeding outcomes.

\section{Material and methods}

\section{Eligibility criteria}

Studies were eligible if participants within the study included older adults with type 2 diabetes - mean age $\geq 55$ years. Studies published from 2000 to the date of the last searches February 25, 2014 - were included. English language only publications were considered. Studies were eligible if they included at least one distinct measure of cognition and at least one distinct measure of self-management and had compared these two measures to report a correlation or other effect size between them.

\section{Information sources}

Four databases were searched separately through the Athens portal: the Cumulative Index to Nursing and Allied Health Literature (Cinahl); Excerpta Medica Database (Embase); Medical Literature Analysis and Retrieval System (Medline); and Psychological Information (PsychInfo). The suitability and appropriateness of the databases chosen were confirmed by an experienced hospital head librarian.

\section{Search}

Each database was searched using the following terms and parameters: "diabetes" and "cognition" or "cognitive", "cognitive impairment", "mild cognitive impairment", "cognitive deficit", "memory", "neuropsychological test", "executive function", and "dementia" and "self-management" and "self-care"; publication year 2000-2014; and English language.

All terms were mapped to the individual thesaurus for each database. All relevant terms were then chosen and exploded to include the full scope of each term.

\section{Study selection}

Studies were screened according to previously stated eligibility criteria. Studies were included that provided effect sizes or correlations, or the means to compute them, for relationships between at least one distinct measure of cognition against at least one distinct measure of self-management.

\section{Risk of bias in individual studies}

Risk of bias was addressed by considering the points in a "Risk of Bias" table from the Cochrane Handbook for Systematic Reviews of Interventions. ${ }^{6}$ Each study was assessed for the following variables, where appropriate in each study:

- selection bias (both random sequence generation and allocation concealment);

- performance bias (blinding of participants and personnel);

- detection bias (blinding of outcome assessment);

- attrition bias (incomplete outcome data);

- reporting bias (selective reporting: eg, of assessment results).

The information gained from assessing risk of bias in the individual studies has been used to inform the suitability and practicalities of data synthesis across studies. Information gained has not been used to create a scale with which to weight studies, however, since research into scales used for this purpose has not shown their validity and has not found improved clarity for readers. ${ }^{6}$ Instead, risks of bias have been presented individually for each study.

Additional quality judgments have been made according to study power (number of participants) and individual characteristics.

\section{Summary measures}

Summary measures varied greatly between studies and included odds ratios (ORs) and $P$-values, Pearson's correlation coefficients, measures score ratios, analysis of covariance 
(ANCOVA) $F$ scores, raw scores, means and standard deviations (SDs), chi-square and Student's $t$-tests, and ratios with confidence intervals (CIs).

\section{Additional analyses}

Heterogeneity across studies was considered to assess the validity of combining study results. Like other similar studies, the small number of studies included in this meta-analysis meant that performing a statistical check for variation across studies was not appropriate. Instead, a judgment about heterogeneity across the included studies was made by assessing differences between studies in a set of key characteristics (sample size and participant characteristics, study design, aim and methods, conclusions, measures of cognition and self-management, effect size statistics between cognition and self-management measures, and potential moderators).

\section{Study selection}

Figure 1 details the study selection process. Four databases were searched through the Athens portal: Cinhal, Embase, Medline, and PsychInfo. Cinahl, indexes more than 3,000 journals relating to nursing and allied health. Embase contains Medline plus over 24 million indexed records and over 7,500 current, mostly peer-reviewed journals. On the advice of an experienced librarian, Medline was also searched separately. PsychInfo is an abstract database that provides systematic coverage of the psychological literature. A total of 1,519 papers were identified (40 through Cinahl, 1,016 through Embase, 156 through Medline, and 307 from PsychInfo). Three hundred and seventy-five were screened and 342 deemed not relevant. Thirty-two studies were further screened and 20 of those did not meet the eligibility criteria. In total, 12 studies were eventually chosen for inclusion into the systematic review. Papers were included if they provided a correlation statistic or other effect size, or the means to compute one, that showed the relationship between one or more cognitive measures and one or more self-management measures. Full papers that were not included after reading were discarded for one of three reasons: unsuitable age of participants (mean age of study participants $<55$ years), failure to include at least one distinct measure of cognition with which to compare a measure of self-management, and failure to include at least one distinct measure of self-management with which to compare a measure of cognition (papers that only assessed glycemic control using glycated hemoglobin $\left[\mathrm{HBA}_{1 \mathrm{c}}\right]$ without an additional specific self-management measure were also excluded).

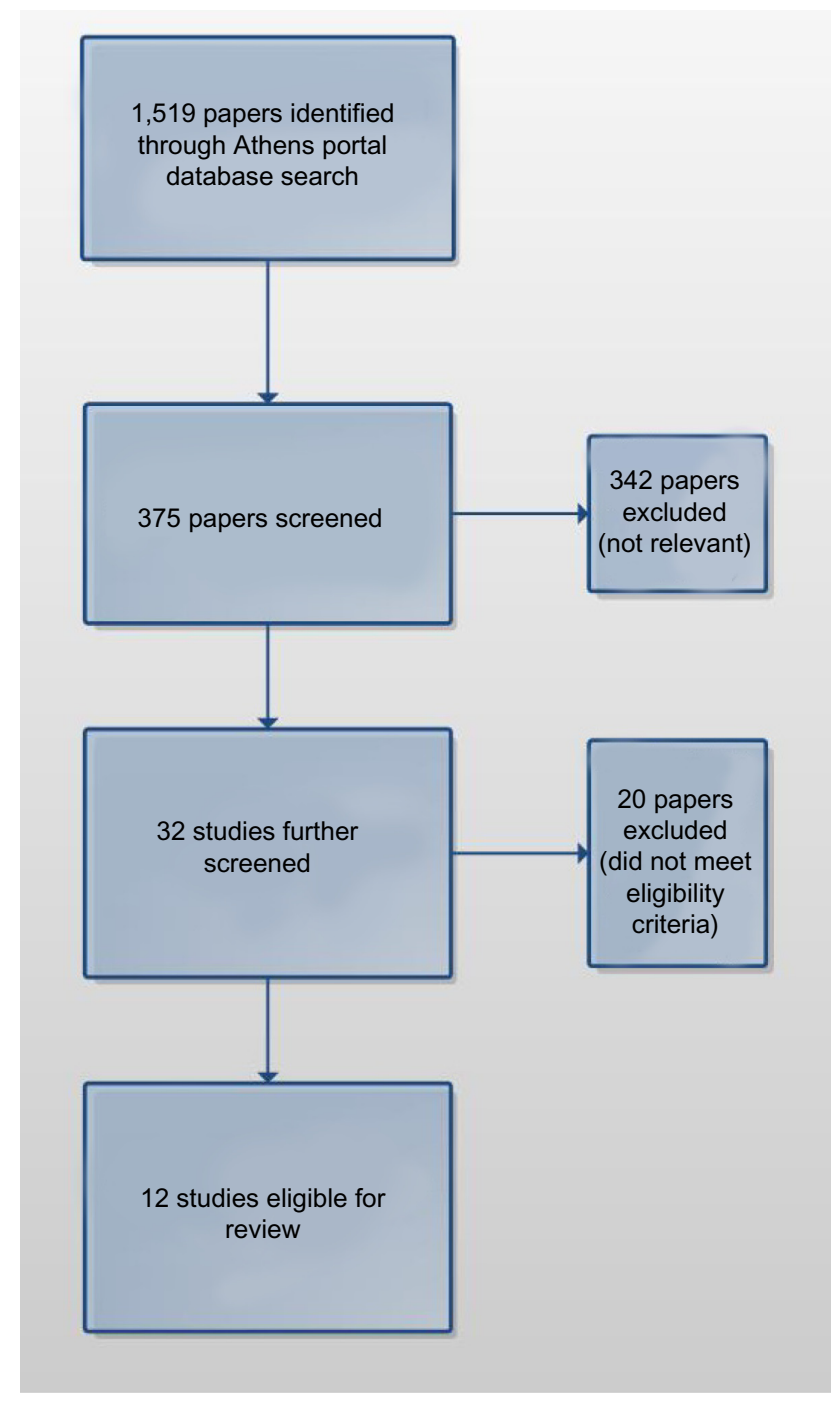

Figure I Eligible studies for review.

Note: Reproduced from Moher D, Liberati A, Tetzlaff J, Altman DG; The PRISMA Group. Preferred reporting items for systematic reviews and meta-analyses: the PRISMA statement. Ann Intern Med. 2009; I5I (4):264-269. ${ }^{28}$

Abbreviation: PRISMA, Preferred Reporting Items for Systematic Reviews and Meta Analyses.

\section{Study characteristics}

Study characteristics are indicated in Table 1, which provides information for each study on sample size, study design, main aims, measures of cognition, and measures of self-management.

\section{Aims and methodology of individual studies}

All studies looked at measures of cognition and measures of self-management in older people with type 2 diabetes. In some studies, investigation of this association was the main aim; in others, it was an additional consideration. 
Table I Study characteristics

\begin{tabular}{|c|c|c|c|}
\hline Study & Study design and main aim & Measure(s) of cognition & Measure(s) of self-management \\
\hline $\begin{array}{l}\text { Cavanaugh } \\
\text { et } \text { al }^{23} \\
\mathrm{~N}=398\end{array}$ & $\begin{array}{l}\text { Cross-sectional survey; aim to examine the } \\
\text { association between diabetes-related } \\
\text { numeracy and glycemic control and other } \\
\text { diabetes measurements }\end{array}$ & $\begin{array}{l}\text { Health literacy (using the Rapid } \\
\text { Estimate of Adult Literacy in } \\
\text { Medicine), general numeracy } \\
\text { (using the Wide Range Achievement } \\
\text { Test, Third Edition), and diabetes- } \\
\text { related numeracy (using the } \\
\text { Diabetes Numeracy Test) }\end{array}$ & $\begin{array}{l}\text { Diabetes knowledge, perceived self- } \\
\text { efficacy of diabetes self-management, } \\
\text { "self-management behaviors", } \mathrm{HBA}_{\mathrm{Ic}}\end{array}$ \\
\hline $\begin{array}{l}\text { Feil et } \mathrm{al}^{16} \\
\mathrm{~N}=5 \mathrm{I}\end{array}$ & $\begin{array}{l}\text { Cross-sectional, longitudinal descriptive study; } \\
\text { aim to examine the role of cognitive } \\
\text { impairment and caregiver support in } \\
\text { diabetes care adherence and glycemic control }\end{array}$ & $\begin{array}{l}\text { Cognitive Abilities Screening } \\
\text { Instrument }\end{array}$ & $\begin{array}{l}\text { SDSCA - a self-report measure of } \\
\text { diabetes self-management, } \mathrm{HBA}_{\mathrm{lc}} \text { levels }\end{array}$ \\
\hline $\begin{array}{l}\text { Feil et } \mathrm{al}^{26} \\
\mathrm{~N}=1,398\end{array}$ & $\begin{array}{l}\text { Cross-sectional observational; aim to } \\
\text { examine effects of cognitive impairment } \\
\text { on self-management }\end{array}$ & $\begin{array}{l}\text { Telephone interview for cognitive } \\
\text { status (modelled on the MMSE) }\end{array}$ & Self-report questionnaire data \\
\hline $\begin{array}{l}\text { Hewitt et } \mathrm{al}^{20} \\
\mathrm{~N}=\mathrm{I}, 047\end{array}$ & $\begin{array}{l}\text { Questionnaire study; aim to examine } \\
\text { knowledge and management of diabetes } \\
\text { by older people }\end{array}$ & MMSE & $\begin{array}{l}\text { Report of recent eye, foot, and dietician } \\
\text { assessment, diabetes knowledge }\end{array}$ \\
\hline $\begin{array}{l}\text { Kazlauskaite } \\
\text { et } \text { al }^{7} \\
\mathrm{~N}=115\end{array}$ & $\begin{array}{l}\text { Cross-sectional; aim to determine accuracy } \\
\text { of self-monitored blood glucose }\end{array}$ & $\begin{array}{l}\text { Digit Symbol Coding Test, Rey } \\
\text { Auditory Verbal Learning Test, } \\
\text { Speed of Information Processing } \\
\text { (Trail Making A), Executive } \\
\text { Function (Trail Making B) }\end{array}$ & Glucose diaries, meter readings, $\mathrm{HBA}_{\mathrm{Ic}}$ \\
\hline $\begin{array}{l}\text { Nguyen et al }{ }^{14} \\
\mathrm{~N}=95\end{array}$ & $\begin{array}{l}\text { Cross-sectional; aim to examine the } \\
\text { association between glycemic control and } \\
\text { executive function and to identify risk factors } \\
\text { that may explain the relationship }\end{array}$ & $\begin{array}{l}\text { Animal Verbal Fluency Test, } \\
\text { Brief Attention Test, Digit Span } \\
\text { Backward Test (from the } \\
\text { Weschler Memory Scale III) }\end{array}$ & $\begin{array}{l}\text { The Michigan Diabetes Research and } \\
\text { Training Center "Diabetes Knowledge } \\
\text { Test" three indicators of self-care } \\
\text { behaviors: } \\
\text { - One question from the diabetes } \\
\text { mellitus module of the Behavioral Risk } \\
\text { Factor Surveillance System (to obtain } \\
\text { blood glucose testing frequency) } \\
\text { - Two questions from the SDSCA }\end{array}$ \\
\hline $\begin{array}{l}\text { Primozic et } \mathrm{al}^{24} \\
\mathrm{~N}=98\end{array}$ & $\begin{array}{l}\text { Cross-sectional; aim to associate specific } \\
\text { cognitive abilities with self-management } \\
\text { behavior }\end{array}$ & $\begin{array}{l}\text { Repeatable Battery for the } \\
\text { Assessment of Neuropsychological } \\
\text { Status }\end{array}$ & SDSCA \\
\hline $\begin{array}{l}\text { Rosen et } \mathrm{al}^{21} \\
\mathrm{~N}=79\end{array}$ & $\begin{array}{l}\text { Correlational; aim to determine whether } \\
\text { neuropsychological function is associated with } \\
\text { adherence to prescribed diabetes medication }\end{array}$ & $\begin{array}{l}\text { MMSE, Trails A and B, Stroop } \\
\text { Color and Word Test, Digit Span, } \\
\text { Digit Symbol, Grooved Pegboard }\end{array}$ & $\begin{array}{l}\mathrm{HBA}_{\mathrm{Ic}} \text {, number of primary care clinic } \\
\text { appointments missed, adherence to } \\
\text { metformin measured with MEMS caps }\end{array}$ \\
\hline Sinclair et al ${ }^{19}$ & Community-based, case-control study; aim & MMSE, CDT & Diabetes knowledge questionnaire \\
\hline
\end{tabular}

$\mathrm{N}=396$ to determine whether cognitive impairment is associated with changes in self-care behavior and use of health and social services in older subjects with diabetes mellitus

Thabit et al ${ }^{10} \quad$ Cross-sectional/correlational; aim to look for $\mathrm{N}=50 \quad$ associations between executive function and selfcare ability in older people with type 2 diabetes

Trimble et $\mathrm{al}^{22} \quad$ Observational; aim to determine if the CDT $\mathrm{N}=30 \quad$ can be used to predict difficulty learning insulin injection in elderly subjects

Vedhara et $\mathrm{al}^{27} \quad$ Correlational; aim to investigate habitual $\mathrm{N}=48 \quad$ prospective memory and medication adherence

Executive Interview 25, Frontal

Assessment Battery, MMSE

CDT, MMSE

Computer-based habitual prospective memory task
SDSCA scale, $\mathrm{HBA}_{\mathrm{Ic}}$

Sham insulin injection skill, $\mathrm{HBA}_{\mathrm{Ic}}$

Electronic monitoring of medication using a medication container with an electronic dose event monitor cap

Abbreviations: $\mathrm{HBA}_{\mathrm{lc}}$, glycated hemoglobin; SDSCA, Summary of Diabetes Self-Care Activities; MMSE, Mini Mental State Examination; MEMS, Medication Event Monitoring System; CDT, Clock Drawing Test; N, number of subjects.

However, correlations between the domains of cognition and self-management in type 2 diabetes remain the focus for this review.

Kazlauskaite et $\mathrm{al}^{7}$ investigated the accuracy of selfmonitored blood glucose in type 2 diabetes in 115 adults with type 2 diabetes recruited from a hospital diabetes clinic. Participants' mean age was 56 years, $63 \%$ were female, and $82 \%$ were taking insulin, with median diabetes duration of 13 years. The study took a cross-section of hospital clinic attendees and investigated blood glucose diaries and 
data downloaded from meters (when provided), alongside measures of literacy, depression, and cognitive function. The authors excluded participants where there was evidence of a neuropsychiatric condition that could influence cognitive assessments, end-stage renal disease, or hospitalizations within the last 30 days. Risks of selection bias were high given the relatively small sample size of 115 and participants drawn from a single institution. Risks of attrition bias were also high, since only half the sample brought their glucose meters to the assessment, meaning that comparisons between reported (diary) and recorded (meter) blood glucose could not be made.

Information in diaries was found to be often inaccurate, and the battery of cognitive tests identified a high prevalence of cognitive dysfunction within the sample. However, the only significant association between the accuracy of blood glucose monitoring reporting and any neuropsychological variables was performance on the Digit Symbol Coding Test $(\mathrm{OR}=2.2, P$-value $=0.02,95 \% \mathrm{CI})$. This is a subtest of the Wechsler Adult Intelligence Scale (WAIS-III), which assesses memory. Performance of the test also involves visual searching and shifting and may be influenced by age or psychomotor slowing. ${ }^{8}$ Three other tests were employed: Rey Auditory Verbal Learning Test (RAVLT), Speed of Information Processing (Trail Making A), and Executive Function (Trail Making B). Deficits in verbal learning ability have been previously associated with type 2 diabetes. ${ }^{9}$ Along with glucose diaries and meter readings, $\mathrm{HBA}_{1 \mathrm{c}}$ levels were also taken. In the subgroup of 50 participants who brought both diaries and meters, the OR of inaccurate reporting increased by 1.3 for each $1 \%$ higher $\mathrm{HBA}_{1 \mathrm{c}}(P=0.08)$. Results may have been mediated by depression; screening for depression using the Beck Depression Inventory - Fast Screen showed that $26 \%$ of respondents had moderate or severe symptoms. The presence of hypertension may also have influenced results. Inaccurate glucose diaries as compared with data downloaded from meters were significantly predicted by normotension (OR 5.6, $P$-value $=0.02$ ).

Kazlauskaite et al's ${ }^{7}$ study was judged to have a high risk of selection bias, since the sample size was relatively small $(\mathrm{N}=115)$, the study was conducted at a single institution, $63 \%$ of participants were female, and $60 \%$ were African American. The risk of attrition bias was also high, since blood glucose meter readings were absent for half the sample after only $50 \%$ of patients brought their glucose meter to the assessment. Reporting bias was deemed to be low, as results for all tests were reported, including those that yielded nonsignificant results.
Another study that explored executive ability alongside diabetes self-care was Thabit et al's ${ }^{10}$ investigation of elderly hospital patients. This research used two measures of executive function to assess cognition: the Frontal Assessment Battery (FAB) and Executive Interview 25 (EXIT 25), alongside two measures of self-care: the Summary of Diabetes Self-Care Activities (SDSCA) scale - a quantitative measure of diabetes self-care over the previous 7 days $^{11}$ - and $\mathrm{HBA}_{1 \mathrm{c}}$ levels. The FAB examines conceptualization, item generation/letter fluency, motor sequencing, susceptibility to interference, inhibitory control, and environmental autonomy. ${ }^{8}$ EXIT 25 assesses similar areas of frontal dysfunction, including verbal fluency and impulse control. ${ }^{12} \mathrm{~A}$ global measure of cognitive function, the Mini Mental State Examination (MMSE), was also included. A cross-section of 50 patients with type 2 diabetes was recruited from a hospital diabetes clinic. Participants were older, with a mean age of 67 years, $68 \%$ were male, and average duration of diabetes was just over 8 years. Cognitive test performance was directly compared with scores on the SDSCA and HBA lc $_{1 \mathrm{c}}$ levels. Mean MMSE score was 27.6. It is generally accepted that a score of $\leq 24$ on the MMSE is indicative of cognitive impairment; ${ }^{8}$ however, both age and level of education are known to influence score. For a participant population with a mean age of 67 years, as in this study, normative mean MMSE scores could range according to education from 22 (those with $0-4$ years of education) to 29 (those educated to college level or higher degree). ${ }^{13}$ Mean EXIT 25 score was 9.5. A higher score is indicative of greater impairment; in this study, $15 \%$ of participants scored $>15$, indicating the presence of impairment. Scores on the FAB measured greater impairment; $48 \%$ of participants had scores indicating impairment. It could be speculated that although both are measures of executive function, FAB and EXIT 25 are measuring slightly different abilities. However, in an investigation comparing the two together, ${ }^{13}$ it was concluded that similar information was provided by both, although the FAB took less time to complete and was more favorable to participants.

Both EXIT 25 and MMSE scores showed significant correlation with $\operatorname{SDSCA}(r=0.03, P=0.04$, and $r=0.5, P<0.05)$. FAB scores did not show a significant association with

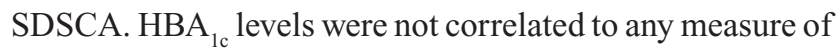
cognitive function. The study identified some cognitive dysfunction in this sample and showed significant associations of self-care, as measured by the SDSCA, with one measure of executive function and one global measure of cognitive function. Success or skills of self-care may therefore be influenced by cognitive dysfunction in this sample. 
The risk of selection bias in Thabit et al's ${ }^{10}$ study was considered to be high, since the sample size was small $(\mathrm{N}=50)$, participants were recruited from one clinic, and the sample had "relatively tight glycemic control and metabolic profile". Attrition bias was low, since the data set was complete. Consent was requested during a hospital consultation, and the assessments were carried out during the same consultation. Reporting bias was low. Nonsignificant results were reported, as were individual test raw scores.

Nguyen at al ${ }^{14}$ conducted a further study investigating links between glycemic control and investigative function, also exploring risk factors that might explain any relationship. Participants were 96 rural Americans with a mean age of 72.2 years, $53 \%$ were female, and average diabetes duration was 14.3 years. Measures of executive function were obtained with three neuropsychological tests: Animal Verbal Fluency Test, Brief Attention Test, and Digit Span Backward Test.

The Animal Verbal Fluency Test is a measure of categorical verbal fluency. One study comparing the effects of age and education on phonemic verbal fluency and animal naming fluency tests found that animal naming was more sensitive to the effects of age; ${ }^{15}$ number of animals generated remained fairly constant up to the age of 60 years, after which score decreased with age.

The Brief Attention Test measures divided attention in verbal-linguistic systems, asking respondents to distinguish and report numbers from a list of letters and numbers read to them, as well as a second test where the participant must disregard the numbers and report the letters. The test is thought to be sensitive to mild cognitive impairment.

Digit Span Backward Test is a subtest of the WAIS-III and involves both visual and verbal processes. It is sensitive to dementia. ${ }^{8}$ Nguyen et al ${ }^{14}$ created a composite score of all three cognitive tests to use as an overall measure of executive function.

Diabetes self-care was assessed using the Michigan Diabetes Research and Training Center "Diabetes Knowledge Test" and three measures of self-care behaviors - one question from the Diabetes Module of the Behavioral Risk Factor Surveillance System (to obtain blood glucose testing frequency) and two questions from the SDSCA (to assess diet and physical activity). The study found executive function to be significantly associated with $\mathrm{HBA}_{1 \mathrm{c}}$ after adjusting for age, sex, education, ethnicity, depressive symptoms, and duration of diabetes (a one-point higher executive function score was associated with a $0.47 \%$ lower $\mathrm{HBA}_{1 \mathrm{c}}$ value $[P=0.01]$ ). This correlation became nonsignificant when all glycemic control risk factors were introduced to a regression analysis
$(P=0.8)$. Glycemic control risk factors were considered to be diabetes knowledge, diabetes medications, and self-care behaviors, suggesting that any cognitive dysfunction found in this population may impact upon these two diabetes selfcare behaviors and skills, and that type of medication may also mediate any effect.

Selection bias in the study by Nguyen et al ${ }^{14}$ was considered medium risk. The sample size was relatively small at 95. Participants were chosen from countries with large minority populations where a high proportion are below the US federal poverty line. However, this was the target population of interest. Additionally, some participants were chosen by "word of mouth" referral and from populations that had previously taken part in studies. Attrition bias was low. Five participants were excluded from the analyses because of missing data. Reporting bias was also low. Nonsignificant results were reported and multivariable regressions performed to quantify the influence of several known modifying factors.

Another study to use the SDSCA as its measure of diabetes self-management, alongside $\mathrm{HBA}_{1 \mathrm{c}}$, was Feil et al's ${ }^{16}$ investigation of the role of cognitive impairment and caregiver support in diabetes management of older outpatients. Fifty-one adult males with type 2 diabetes were recruited from a geriatric clinic at a veterans' health care center; mean age was 78 years and duration of diabetes was at least 6 months. This study is unusual among those within this review, since it was also designed to assess the influence of caregiver support in diabetes management. The authors employed the Cognitive Abilities Screening Instrument (CASI) to assess cognitive function. The measure includes tasks assessing attention, concentration, orientation, memory, language, visual construction, verbal fluency, abstract reasoning, and judgment. The CASI has been used to detect dementia; ${ }^{17}$ one study that looked at data from cognitively intact older people undertaking the test found it to be influenced, like other cognitive tests, by age and education. ${ }^{18}$ Diabetes self-care was measured using the SDSCA.

The study found that participants with cognitive impairment were more likely to report worse adherence to their diabetes care (ANCOVA $F=5.1, P<0.5$ ). Caregiver involvement may have obscured some potential correlations between cognitive impairment and self-care, since either patient or caregiver could complete the SDSCA. Depression may also have mediated some of the cognitive effect. Participants with depression were more likely to report worse adherence (ANCOVA $F=7.9, P<0.001$ ). 
Selection bias was high in the study by Feil et al, ${ }^{16}$ given a small sample size of 51 recruited from one veterans' clinic. There were some incomplete data. Three $\mathrm{HAB}_{1 \mathrm{c}}$ levels were missing at baseline, and the risk of attrition bias was high. At 1-year follow-up, a further nine $\mathrm{HBA}_{1 \mathrm{c}}$ levels were missing, although the authors state that five patients without $\mathrm{HBA}_{1 \mathrm{c}}$ levels were deceased, and there were no differences in missing data between those with caregivers and those without. Reporting bias was low. Insignificant results were reported and subgroups were statistically investigated.

Sinclair et $\mathrm{al}^{19}$ investigated cognition dysfunction in older subjects with diabetes in relation to self-management. This was the only study in this review selection that directly considered impacts of cognitive dysfunction on use of care services. Participants were registered with general practitioner (GP) surgeries in Wales and included 396 adults (51\% female) with a mean age of 74.9 years and a mean diabetes duration of 10 years. Ninety-five percent of participants had type 2 diabetes - this was one of only two studies in this review to include any participants with type 1 diabetes - and $17.55 \%$ were treated with insulin. This was a case control study with 393 age- and sex-matched nondiabetic controls.

The aim was to determine whether cognitive impairment was associated with changes in self-care behavior and use of health and social services in older people with diabetes, and the outcome measures were adjusted OR estimates of normal performance on the MMSE and Clock Drawing Test (CDT), alongside information on self-care behaviors and use of services. In comparison with diabetic participants with no evidence of cognitive impairment, diabetic participants with an MMSE score of $<23$ were significantly less likely to be involved in diabetes self-care $(P=0.0001)$ and diabetes monitoring $(P=0.001)$. In this study, self-care measures relied on self-report and assessed levels of responsibility for self-care rather than specific self-care skills or behaviors.

Selection bias was considered to be medium risk in Sinclair et al's ${ }^{19}$ study. No randomization was reported. Participants were identified through GP records, although $83 \%$ of eligible subjects completed the study. Attrition bias for incomplete outcome data was considered to be at medium risk, since diabetes treatment was not reported in three cases. There was a low risk of reporting bias, since nonsignificant results were reported.

Another study to employ the MMSE was Hewitt et al's ${ }^{20}$ investigation of self-management and patient understanding of diabetes in the older person. Similarly to the previous study, the authors also used the MMSE cut-off score of $\leq 23$ to indicate cognitive impairment. Self-management was assessed by reports of attending recent eye, foot, and dietician assessments and by gathering responses to questions of diabetes knowledge, including, for those taking insulin, responses to low blood sugar and sick day rules questions and, for all participants, questions on diabetes treatment and use of health care services.

Participants using insulin and with cognitive impairment were significantly more likely not to know what to do in the event of low blood sugar or how to manage medication in the event of sickness. In this study, metabolic parameters may have had an influence. Twelve people had self-reported diabetes unconfirmed by any health professionals. Moderating factors were considered. Social deprivation was explored and made no difference to self-care measures except one; people from lower socioeconomic groups gave more incorrect responses regarding taking a snack in the presence of hypoglycemia $(P=0.003)$. Risks of selection bias were low. One hundred and six general practices from the Medical Research Council General Practice Research Framework, selected to be representative of the UK distribution of mortality and deprivation (Jarman scores - a measure of general practice workload that has been used by the Department of Health to determine additional payments to GPs considered to practice in deprived areas), were randomized to two health assessments and further randomized to two care teams. There was some risk of attrition bias, since $7.2 \%$ of participants did not respond to a question on the frequency of home glucose testing, but these participants were still included in some analysis.

Judging selection bias was not relevant, since there was no direct intervention. Data were gained through report of a questionnaire study (although questionnaire data were derived from an intervention study). There were some incomplete data: eg, $7.2 \%$ of participants did not respond to the question on the frequency of home glucose testing, but those participants were still included in some analysis. Reporting bias was low risk, since scores for each domain were reported.

One study looked at a very specific association between cognition and diabetes self-care, investigating cognition in relation to correct adherence to metformin. ${ }^{21}$ Similarly to Feil et al's ${ }^{16}$ study, the participant group for this study was recruited from a veterans' clinic. This group may be composed of more frail individuals and was $100 \%$ male. Average age was 65 years, with a range of $41-85$ years. MMSE was used as a screening tool, with those participants scoring $<23$ on the measure considered to have severe cognitive impairment and subsequently excluded from the study. 
The main aim of the research was to determine whether neuropsychological function was associated with adherence to prescribed diabetes medication. Patient medication information was solicited and confirmed by health professionals involved in treating the participants. $\mathrm{HBA}_{1 \mathrm{c}}$ was analyzed, and appointment-keeping records were collected from computerized records.

Five neuropsychological tests were administered: Trails A and B (timed tracking tasks employing visual and motor systems that are sensitive to executive function deficits), Stroop Color and Word Test (a test of selective attention), Digit Span and Digit Symbol from the WAIS (measure working memory and attention, respectively, and complex psychomotor functioning), and Grooved Pegboard (another visuomotor test involving hand-eye coordination).

Adherence to prescribed diabetes mediation was assessed by placing MEMS caps on participants' metformin bottles. MEMS caps have embedded microchips that record data regarding bottle opening incidence. Measurements were taken over a 4-week period. An adherence figure was derived by taking the percentage of doses taken within 2 hours of the agreed upon dose time, divided by the number of doses prescribed.

Stroop word score was significantly associated with adherence $(\rho=0.26, P<0.05)$, time to complete Trail B was negatively correlated with adherence $(\rho=-0.26, P<0.05)$, and low scores on the MMSE were associated with missed clinic appointments. No neuropsychological variable was associated with $\mathrm{HBA}_{1 \mathrm{c}}$.

Potential moderating variables were investigated. Age was significantly associated with adherence, and non-Caucasian ethnicity was associated with missed appointments. Years of education were not considered, and insulin use was not reported.

Rosen et $\mathrm{al}^{21}$ had a relatively small sample size at 79 recruited participants from one clinic with a $100 \%$ male sample, and there was no random sequence generation, so selection bias was judged to be high for this study. No missing data or attrition over the 4-week period of observation were reported, so we were unable to make a judgment about the risk of attrition bias for incomplete outcome data for this study. There was a low risk of reporting bias, since raw scores were presented and nonsignificant results reported.

One study investigated insulin administration skills in older adults alongside cognition. Trimble et $\mathrm{al}^{22}$ recruited 30 insulin-naïve adults with type 2 diabetes using oral antihyperglycemic agents for their observational study. Mean age was 77 years with an SD of 1 year, $53.3 \%$ of participants were female, and average diabetes duration was 12 years. Participants were excluded if there was a history of cerebrovascular accident resulting in neurological disability, poor eyesight, poor hearing, non-English speaking, or any previous insulin use. The study's main aim was to determine if the CDT (as also employed in Sinclair et al's ${ }^{19}$ study) could be used to predict difficulty learning the skills of insulin injections in older subjects. The CDT is a measure of global cognition and is quick and simple to administer. The MMSE was also employed in this study. Here the authors report using a cut-off score of $<27$ to denote evidence of a cognitive deficit, as generally used for those with a higher level of education.

Self-care was measured by performance of a skill learnt during the study (performance was scored after 7 days of practice): a sham insulin injection with an insulin pen using a standardized protocol. Skills were scored as correct, minor problems (would not result in inaccurate insulin dosing), or major problems (would result in inaccurate dosing). $\mathrm{HBA}_{1 \mathrm{c}}$ levels were also recorded.

Both cognitive tests found some associations with skill at performing the sham insulin injections. Subjects with an abnormal score on the CDT were significantly more likely to have difficulty learning to safely administer a sham insulin injection ( $P=0.01$, chi-square test). An abnormal MMSE (a score of $<27$ ) did not predict difficulties with the sham insulin injection or incorrect needle count, but it was associated with a longer piston length $(P<0.05$, Student's $t$-test). Selecting length is one of the measured stages in performing the sham insulin injection.

Moderators were unreported. The effects of potential comorbidities or socioeconomic variables were unexplored. There was a small sample size of only 30 participants, creating a high risk of selection bias. Also, participants were recruited from the same hospital clinic; the authors make no mention of randomized selection procedures. Participants attended the clinic three times during the course of the study, where a registered nurse who was blinded to the results of the first visit conducted the second and third visits. All participants completed the CDT, but those who could not safely follow all steps on the sham insulin instruction sheet did not proceed to visit three; thus, the study may have excluded some participants with existing cognitive or psychomotor deficits.

Trimble et $\mathrm{al}^{22}$ had the smallest sample size of all eligible studies, making the risk of selection bias high. Risk of performance bias (blinding of participants and researchers) was deemed to be low, since a registered nurse who was 
blinded to the results of the first visit conducted the second and third visits. There was a medium risk of incomplete data. All subjects completed the CDT. Those attending the clinic with a blood glucose test of $<4.0 \mathrm{mmol} / \mathrm{L}$ were discontinued from the study and removed from the analysis. Subjects who could not safely follow all steps on the sham insulin instruction sheet did not proceed to visit three. Twenty-eight subjects completed all three visits and one was removed from the analysis. Reporting bias was low risk, since nonsignificant results were reported.

One further study to have investigated cognition alongside self-care is Cavanaugh et al's ${ }^{23}$ study of the association of numeracy and diabetes control. Skill in numeracy is both a fundamental everyday cognitive ability and a key skill in diabetes, especially for those who are prescribed insulin. Doses must be measured and adjusted, and carbohydrates must be counted. Cavanaugh et $\mathrm{al}^{23}$ recruited 398 adult patients with type 1 or type 2 diabetes from two primary care and two diabetes clinics at three medical centers. Median age of participants was 55 years, with a range of 46-64 years, 51\% were female, and 86\% had type 2 diabetes. Median duration of diabetes was 9 years, and $61 \%$ were taking insulin.

The authors' main aim was to examine the association between diabetes-related numeracy and glycemic control and other diabetes measurements. Both general numeracy and diabetes-specific numeracy were assessed. General numeracy was measured with the Wide Range Achievement Test, Third Edition, and diabetes-related numeracy with the Diabetes Numeracy Test (DNT). Patient performance was calculated as a percentage of questions answered correctly. To assess self-care, participants were given the Diabetes Knowledge Test, the Perceived Diabetes Self-Management Scale, and the SDSCA.

The study found poor numeracy skills to be common in participants. Low scores on the specific measure of diabetesrelated numeracy were associated with worse perceived selfefficacy and fewer self-management behaviors. DNT score was also associated with $\mathrm{HBA}_{1 \mathrm{c}}$ levels, suggesting that DNT influences self-management behaviors that, in turn, have an effect upon $\mathrm{HBA}_{1 \mathrm{c}}$ levels.

Cavanaugh et $\mathrm{al}^{23}$ reported no randomization procedures in their selection procedure, increasing the risk of selection bias. Although eight participants did not complete the study and two participants were missing $\mathrm{HBA}_{1 \mathrm{c}}$ scores, risks associated with incomplete data were considered low, since "a sensitivity analysis based on multiply imputed data gave similar results for the association between DNT score and
$\mathrm{HBA}_{1 \mathrm{c}}{ }^{\prime}$. Raw scores for all assessments were reported, making the risk of reporting bias low.

Primozic et $\mathrm{al}^{24}$ investigated diabetes self-management and cognitive ability in a cross-sectional study with 98 adults attending a diabetes outpatient clinic in Slovenia. Participants had had type 2 diabetes for at least 2 years, $61.2 \%$ had an insulin prescription, and their average age was $63.74( \pm 9.87)$ years. Diabetes self-management ability was assessed using the SDSCA. ${ }^{11}$ Cognition (immediate and delayed memory, attention, language, and visuospatial/ constructional abilities) was measured using the Repeatable Battery for the Assessment of Neuropsychological Status (RBANS). ${ }^{8}$ Interestingly, the authors also measured various domains of emotion (depression and diabetes-related distress) to see if these moderated any cognitive effect on self-management. The authors noted that better executive functions were the strongest predictor of better diabetes self-management $(P<0.042)$, although executive function scores also correlated with RBANS total score and attention subtest. In multivariate regression, this association was independent from depression (as measured by the Hamilton Depression Inventory). ${ }^{25}$ Risks of selection bias were high, since the study was relatively low powered, and participants were all recruited at the same outpatient clinic.

Feil et $\mathrm{al}^{26}$ conducted a cross-sectional observational analysis of 1,398 older adults (mean age 70 years, $\mathrm{SD}=7.4$ ) with type 2 diabetes to investigate links between cognition and diabetes self-management in this population. Five self-management domains (medication, exercise, diet, blood glucose checking, and foot care) were measured using the Diabetes Care Profile. Cognition was measured with the Telephone Interview for Cognitive Status (modeled on the MMSE). As a telephone tool, this necessarily omits assessments for visual perception and memory and psychomotor function; the latter cognitive function has been implicated in previous studies as particularly affected in people with type 2 diabetes, so this cognitive tool may not have captured all cognitive impairment in this group. Like other studies, self-management domains were self-reported. Compared with the least cognitively impaired, participants who showed greater cognitive impairment were less likely to be able to exercise regularly and were also less likely to follow recommended eating plans. Links between cognitive ability and checking blood sugar levels or feet were not demonstrated. Those with worse diabetes comorbidities were less likely to be able to exercise regularly (OR $=0.962$, $P<0.01$ ); this association was weaker for ability to follow eating plans $(\mathrm{OR}=0.969, P<0.01)$, suggesting that poorer 
cognition may worsen dietary management above the negative effect of greater comorbidities.

Vedhara et $\mathrm{al}^{27}$ investigated a very specific cognitive domain - habitual prospective memory (HPM) - and its association with medication adherence in 48 older individuals identified through three primary care practices. HPM was measured using a computerized task. Adherence to oral antidiabetic medication was measured electronically using an electronic dose event monitor cap, which records the date and time that the container is opened. The authors found that overall task performance was related to medication adherence, although the nature of errors made on the task (forgetting to complete a task or completing a task too soon or too late) was not. The results suggest that in older individuals, deficits in HPM - being able to repeatedly remember to take an action in the future - may be a useful indicator for difficulties with medication adherence. The authors excluded those with cognitive impairment from the study, however, which may limit the generalizability of these results to an older population with type 2 diabetes.

\section{Results}

\section{Synthesis of results}

Great heterogeneity across studies on multiple factors made this review unsuitable for meta-analysis. Effects found in the individual studies were not similar enough to estimate a combined effect that would give a meaningful description of the group of studies. Although each study was chosen because it contained at least one measure of cognition and at least one measure of self-management, the disparate focus and main aims between studies had great impact. For example, one study's ${ }^{22}$ use of a sham insulin injection as an outcome measure required participants to be insulin naïve, in direct contrast to other studies that gave figures for the numbers of participants on insulin therapy. ${ }^{7,19,20,23}$ Overall study aims included investigations into the accuracy of self-monitored blood glucose, ${ }^{7}$ specific investigation of associations between executive function and self-care ability in older people with type 2 diabetes ${ }^{10}$ and executive function and glycemic control, ${ }^{14}$ numeracy and glycemic control, ${ }^{23}$ an examination of the role of cognitive impairment and caregiver support in diabetes care adherence, ${ }^{16}$ neuropsychological function and medication adherence, ${ }^{21}$ cognitive impairment, self-care and use of diabetes services, ${ }^{19}$ diabetes understanding, ${ }^{20}$ and cognition and insulin injection proficiency. ${ }^{22}$

Methods of recruitment also reflected great heterogeneity with some studies. Exclusion criteria varied tremendously; some studies explicitly screened for neuropsychological disorders $^{7}$ or neuropsychological disability ${ }^{22}$ and one for low scores on the MMSE. ${ }^{21}$ No studies excluded on the basis of all diabetes complications, although one excluded participants if they had end-stage renal disease. ${ }^{7}$

Although many studies were similar in design, with only one study reporting a control group, ${ }^{19}$ there was a huge variation in sample size, with some studies carrying little power. ${ }^{10,16,22}$

One reason for undertaking a meta-analysis, though unsuitable here due to great heterogeneity across studies, is to synthesize the results from a group of small studies whose results alone do not have much statistical power. Although eight studies in this set had relatively small sample sizes, four studies had relatively large sample sizes, ranging from 396 participants to 1,398 participants, giving their results greater statistical power.

Total sample size across studies was 3,805. Ten studies had one group of participants, ie, no control group, and looked for correlations within participants between measures of self-management and measures of cognition. One study looked at these measures alongside the presence of a caregiver; ${ }^{16}$ this may have obscured any correlations between self-management and cognitive domains in this study, since either patient or caregiver could complete the self-management assessment. Therefore, the results of this study would not be generalizable to a wider population of type 2 diabetes. For participants across all studies, 46.2\% were female. Two studies were $100 \%$ male.

\section{Risk of bias across studies}

Across studies there is a risk of publication bias, since no studies were included from "gray literature" such as unpublished data and conference abstracts, although all studies reported nonsignificant findings. Many sample sizes were small. Median sample size was 96.5 (ranging from 30 to 1,398). Two studies recruited participants from US clinics for veterans - potentially a particularly frail group and most studies did not report formal structured randomized selection procedures.

Most studies considered a wide range of moderating variables in their analysis, with several performing factor or regression analysis to tease out relationships between variables.

\section{Summary of evidence}

Three studies reported excess cognitive dysfunction in their participants. ${ }^{7,10,23}$ Executive function was chosen as a focus for cognitive assessment in four studies. Thabit et a ${ }^{10}$ employed 
EXIT 25 and FAB, and Kazlauskaite et $\mathrm{al}^{7}$ and Rosen et $\mathrm{al}^{21}$ chose to measure executive function with Trail Making B Test from the WAIS. Nguyen et $\mathrm{al}^{14}$ created a composite score from three cognitive tests, Animal Verbal Fluency Test, Brief Attention Test, and Digit Span Backward Test, to use as an overall measure of executive function.

Other neuropsychological tests employed were the Digit Symbol Coding Test from the WAIS-III to assess memory, ${ }^{7,21}$ the RAVLT, and Speed of Information Processing. ${ }^{7}$ Trimble et $\mathrm{al}^{22}$ and Sinclair et $\mathrm{a}^{19}$ used the CDT, a measure of global cognition. Feil et $\mathrm{al}^{16}$ employed the CASI, which assesses attention, concentration, orientation, short-term memory, long-term memory, language abilities, visual construction, list-generating fluency, abstraction, and judgment. Rosen et $\mathrm{al}^{21}$ used a selection of different neuropsychological assessments, including Trail A, Stroop Color and Word Test, Digit Span from the WAIS, complex psychomotor functioning, and Grooved Pegboard. Cavanaugh et $\mathrm{al}^{23}$ assessed a specific area of cognition using the DNT. Five studies used the MMSE, ${ }^{10,19-22}$ one as a screening tool. ${ }^{21}$

The SDSCA was the single most common choice for assessing self-care and was used by five studies. Four used the complete assessment, ${ }^{10,16,23,24}$ and one, Nguyen et al, ${ }^{14}$ used two questions from the scale to assess diet and physical activity.

Other self-care assessment tools included the Michigan Diabetes Research and Training Centre "Diabetes Knowledge Test" and one question from the Diabetes Module of the Behavioral Risk Factor Surveillance System (to obtain blood glucose testing frequency). ${ }^{14}$ In the study by Hewitt et al, ${ }^{20}$ selfmanagement was assessed by reports of attending recent eye, foot, and dietician assessments and by gathering responses to questions of diabetes knowledge, including, for those taking insulin, responses to low blood sugar and sick day rules questions, and, for all participants, questions on diabetes treatment and use of health care services. Rosen et $\mathrm{al}^{21}$ used adherence to prescribed diabetes medication, as measured by MEMS caps microchips, to assess tablet-taking accuracy. Cavanaugh et $\mathrm{al}^{23}$ employed both the Diabetes Knowledge Test and the Perceived Diabetes Self-Management Scale.

Glycemic control was also considered. $\mathrm{HBA}_{1 \mathrm{c}}$ was found to be correlated with inaccurate reporting of blood glucose monitoring in Kazlauskaite et al's ${ }^{7}$ study. Cavanaugh et $\mathrm{al}^{23}$ found that scores on the DNT were associated with $\mathrm{HBA}_{1 \mathrm{c}}$ levels. Nguyen et $\mathrm{al}^{14}$ reported executive function to be significantly associated with $\mathrm{HBA}_{1 \mathrm{c}}$ after adjusting for age, sex, education, ethnicity, depressive symptoms, and duration of diabetes, although this correlation became nonsignificant when all glycemic control risk factors were introduced to a regression analysis. Glycemic control risk factors were considered to be diabetes knowledge, diabetes medications, and self-care behaviors.

Both Thabit et $\mathrm{al}^{10}$ and Rosen et $\mathrm{al}^{21}$ reported no correlation between $\mathrm{HBA}_{1 \mathrm{c}}$ levels and any cognitive variable.

All studies reported direct associations between measures of cognition and measures of self-management with the exception of Nguyen et al, ${ }^{14}$ who had considered self-care variables with cognition only as moderators to the association between cognition and $\mathrm{HBA}_{1 \mathrm{c}}$.

Cavanaugh et $\mathrm{al}^{23}$ found that low scores on the specific measure of diabetes-related numeracy were associated with worse perceived self-efficacy and fewer self-management behaviors. Hewitt et $\mathrm{al}^{20}$ showed that participants using insulin and with cognitive impairment were significantly more likely not to know what to do in the event of low blood sugar or how to manage medication in the event of sickness. Feil et al ${ }^{16}$ concluded that participants with cognitive impairment were more likely to report worse adherence to their diabetes care. Thabit et $\mathrm{al}^{10}$ found that both EXIT 25 and MMSE scores showed significant correlation with SDSCA, although FAB scores did not show any significant association with SDSCA. Trimble et $\mathrm{al}^{22}$ reported that subjects with an abnormal score on the CDT were significantly more likely to have difficulty learning to safely administer a sham insulin injection. Sinclair et $\mathrm{al}^{19}$ noted that in comparison with diabetic participants with no evidence of cognitive impairment, diabetic participants with an MMSE score of $<23$ were significantly less likely to be involved in diabetes self-care and diabetes monitoring.

Rosen et $\mathrm{al}^{21}$ found that Stroop word score, a test of selective attention, and Trail B, a measure of executive function, were significantly associated with adherence, and low scores on the MMSE were associated with missed clinic appointments. Kazlauskaite et $\mathrm{al}^{7}$ reported that scores on the Digit Symbol Coding Test (a subtest of the WAIS-III, which assesses memory) were significantly correlated with inaccuracies in blood glucose reporting, but that measures of verbal learning (RAVLT), speed of information processing, and executive function (Trail Making B) had no significant correlation with blood glucose reporting (Table 2).

\section{Discussion}

\section{Limitations}

The nature of the subjects studied was variable in terms of their disease duration, previous medical histories, associated medical comorbidities, and prior diabetes educational 


\section{Table 2 Summary of results}

Excess cognitive function reported in three studies ${ }^{7,10,23}$

Executive function chosen as focus for cognitive assessment in four studies, $8,11,15,22$

- Executive function significantly associated with adherence ${ }^{21}$ MMSE used as an assessment or screening tool in five studies 10,19-22 $^{21}$

- Low scores on MMSE associated with missed clinic appointments ${ }^{21}$

- MMSE score of $<23$ less likely to perform diabetes self-care and monitoring activities 19

SDSCA was the single most common choice of self-management assessment tool in five studies ${ }^{10,14,16,23,24}$

- Exit Interview 25 and MMSE scores significantly correlated with SDSCA $^{10}$

Significant associations found

- Poor diabetes-related numeracy associated with worse perceived selfefficacy and fewer self-management behaviors ${ }^{23}$

- Participants using insulin and with cognitive impairment less likely to manage low blood sugar events and medication when sick ${ }^{20}$

- Participants with cognitive impairment more likely to report worse adherence to diabetes care ${ }^{16}$

- Abnormal CDT score associated with difficulty learning sham insulin injection procedure ${ }^{22}$

Studies using self-report methods to assess diabetes self-management: $75 \%$

Abbreviations: MMSE, Mini Mental State Examination; SDSCA, Summary of Diabetes Self-Care Activities; CDT, Clock Drawing Test.

attainment. The majority of studies were of an associational nature and not findings confirmed by repeat testing or by the effects of an intervention. The majority of studies were not designed to give a view or conclusion on the clinical value or implications of the research. This only allows speculation on their importance. The domains of diabetes self-management examined are based on health professional- or investigator-led views/opinions as to what is most important in achieving optimal efficiency in diabetes self-care, but may not be the most important as identified by patients or carers. This emphasizes the importance of seeking these views by other methods. It should be remembered that most studies do not separate out the influence of aging itself in altering diabetes self-care behavior, and this requires attention in more in-depth studies.

A further limitation is associated with the difficulties of measuring diabetes self-management skills and abilities. The majority of studies reported here used methods of self-management skills assessment that relied upon selfreport (eg, questionnaires). Only three studies used methods of self-management assessments that allowed objective measurement. Rosen et $\mathrm{al}^{21}$ used MEMS caps to measure medication adherence, Trimble et $\mathrm{al}^{22}$ assessed insulin injection skills using a sham insulin injection protocol, and Vedhara et $\mathrm{al}^{27}$ also used electronic monitoring to assess medication adherence. The remaining studies all relied on self-report to assess self-management behavior.

\section{Conclusion}

Overall, studies in this review have identified deficits in many cognitive areas, but particularly in executive function and memory, and low scores on measures of global cognitive functioning that have shown significant correlations with multiple areas of diabetes self-management, including diabetes-specific numeracy ability (both a measure of cognition and a diabetes self-management skill), diabetes knowledge, insulin adjustment skills, ability to learn to perform insulin injections, worse adherence to medications, decreased frequency of self-care activities, missed appointments, decreased frequency of diabetes monitoring, and increased inaccuracies in reporting blood glucose monitoring.

It is easy to see the potential threats that cognitive impairment could place on diabetes self-care outcomes. Additionally, most studies that investigated moderating variables identified age as an influence on both cognition and self-management. Studies in this review all focused on older people; significant correlations between cognition and self-management in this group may well be exacerbated by age. Clinically, therefore, it is important to note that this vulnerable group may be particularly at risk from the negative effects on self-management brought about by cognitive impairment.

It should also be noted that one study ${ }^{7}$ that screened for depression showed that $26 \%$ of respondents had moderate or severe symptoms. In another study ${ }^{16}$ in this review, a correlation was reported between the presence of depression and reported worse adherence to diabetes care. These findings also have clinical interest in this already vulnerable group. Depression is more common in diabetes and may exacerbate or be mistaken for the symptoms of mild cognitive impairment. Depression could also directly influence self-management outcomes, perhaps through an effect on motivation to self-care.

\section{Clinical implications}

This review has demonstrated that in studies that have examined the relationship between mental performance and diabetes self-management ability in a heterogeneous group of older subjects with type 2 diabetes, multiple significant associations can be demonstrated. The consistency of some observations implies the presence of real associations, although these can only be proved in prospective, intervention-type studies. This review is unable to assign a "clinical" value to the importance of these associations, but it is justified to assume that significant deficits in memory or executive function are likely to 
influence medication adherence, responding appropriately to glucose monitoring values or to hypoglycemia, and taking the right action during sickness. It is also justified to assume that significant impairment of psychomotor function combined with global deficits could lead to an impairment in insulin administration skills.

This review has demonstrated that there is no consensus on methods to examine either global or specific cognitive domains in subjects with diabetes and declining cognition. This increases the difficulty in interpreting the outcomes or judging what is important. In the same vein, there is no consensus on what aspects of diabetes self-management are the most important and have the highest priority.

A decline in mental performance in a patient with diabetes can have wider implications than affecting diabetes self-management skills. By promoting poor glycemic control, it can potentially influence the risk of future vascular disease, both micro- and macroangiopathic in nature. By increasing the risk of worse metabolic control (eg, higher blood pressure levels) or increasing the risk of hypoglycemia, the risk of further cognitive decline may also be exacerbated.

\section{Future studies}

This review clearly supports the conclusion that further research in this area is required. As always, detailed literature reviews and systematic reviews demonstrate a need for more homogeneity and standardization in both the methods used to measure variables and the patient population to be studied. This is not always straightforward. In this review, we have tried to examine a variety of methods, both pencil and paper- and computer-based, to explore this aspect. We have looked at patients with type 2 diabetes, generally those of an older population, where cognitive change may have more impact and influence outcome.

Future studies in this area require more thought on methods to be used (providing a clear pathophysiological reason for including them) and identifying a priority list of diabetes self-care management activities that have a high chance of influencing clinical outcome.

Additional areas that should be considered for further evaluation include:

- developing a cognitive assessment tool for subjects with diabetes that can be used easily and quickly in routine clinical practice as part of screening for cognitive change at diagnosis and for inclusion in the annual review;

- evaluating the costs of problems with diabetes self-care behavior influenced by cognitive change as evidenced by hospital admission rates, ambulance call-out costs for hypoglycemia, and GP visits;

- the role of treatment strategies/interventions that can target either mental performance or diabetes self-care behavior (or both) in maintaining as high a level of diabetes self-management as possible for as long as possible.

\section{Disclosure}

The authors report no conflicts of interest in this work.

\section{References}

1. Skyler JS. Effects of glycemic control on diabetes complications and on the prevention of diabetes. Clin Diabetes. 2004;22:162-166.

2. Velayudhan L, Poppe M, Archer A, Proitsi P, Brown RG, Lovestone S. Risk of developing dementia in people with diabetes and mild cognitive impairment. Br J Psychiatry. 2010;196:36-40.

3. Kodl KT, Seaquist ER. Cognitive dysfunction and diabetes mellitus. Endocr Rev. 2008;29:494-511.

4. Köhler M, Kliegel M, Kaduszkiewicz H, et al. Effect of cardiovascular and metabolic disease on cognitive test performance and cognitive change in older adults. J Am Geriatr Soc. 2012;60:1286-1291.

5. Austin M-P, Mitchell P. Cognitive deficits in depression: possible implications for functional neuropathology. $\mathrm{Br} J$ Psychiatry. 2001;178:200-206.

6. Higgins JPT, Green S, editors. Cochrane Handbook for Systematic Reviews of Interventions. Version 5.1.0. The Cochrane Collaboration, 2011. Available from: http://www.cochrane-handbook.org. Accessed September 12, 2014.

7. Kazlauskaite R, Soni S, Evans AT, Graham K, Fisher B. Accuracy of self-monitored blood glucose in type 2 diabetes. Diabetes Technol Ther. 2009;11:385-392.

8. Lezak M, Howieson DB, Loring DW. Neuropsychological Assessment. 4th ed. New York: Oxford University Press; 2004.

9. Gradman TJ, Laws A, Thompson LW, Reaven GM. Verbal learning and/or memory improves with glycemic control in older subjects with non-insulin-dependent diabetes mellitus. $J$ Am Geriatr Soc. 1993;41:1305-1312.

10. Thabit H, Kennelly SM, Bhagarva A, et al. Utilization of Frontal Assessment Battery and Executive Interview 25 in assessing for dysexecutive syndrome and its association with diabetes self-care in elderly patients with type 2 diabetes mellitus. Diabetes Res Clin Pract. 2009;86:208-212.

11. Toobert DJ, Hampson SE, Glasgow RE. The Summary of Diabetes Self-Care Activities measure: results from 7 studies and a revised scale. Diabetes Care. 2000;23:943-950.

12. Crum RM, Anthony JC, Bassett SS, Folstein MF. Population-based norms for the Mini-Mental State Examination by age and educational level. JAMA. 1993;269:2386-2391.

13. Moorhouse P, Gorman M, Rockwood K. Comparison of EXIT-25 and the Frontal Assessment Battery for evaluation of executive dysfunction in patients attending a memory clinic. Dement Geriatr Cogn Disord. 2009;27:424-428.

14. Nguyen HT, Grzywacz JG, Arcury TA, et al. Linking glycemic control and executive function in rural older adults with diabetes mellitus. J Am Geriatr Soc. 2010;58:1123-1127.

15. Tombaugh TN, Kozak J, Rees L. Normative data stratified by age and education for two measures of verbal fluency: FAS and animal naming. Arch Clin Neuropsychol. 1999;14:167-177.

16. Feil DG, Pearman A, Victor T, et al. The role of cognitive impairment and caregiver support in diabetes management of older outpatients. Int J Psychiatry Med. 2009;39:199-214. 
17. Teng EL, Hasegawa K, Homma A, et al. The Cognitive Abilities Screening Instrument (CASI): a practical test for cross-cultural epidemiological studies of dementia. Int Psychogeriatr. 1994;6: 45-58.

18. McCurry SM, Edland SD, Teri L, et al. The Cognitive Abilities Screening Instrument (CASI): data from a cohort of 2524 cognitively intact elderly. Int J Geriatr Psychiatry. 1999;14:882-888.

19. Sinclair AJ, Girling AJ, Bayer AJ. Cognitive dysfunction in older subjects with diabetes mellitus: impact on diabetes self-management and use of care services. All Wales Research into Elderly (AWARE) Study. Diabetes Res Clin Pract. 2000;50:203-212.

20. Hewitt J, Smeeth L, Chaturvedi N, Bulpitt CJ, Fletcher AE. Self-management and patient understanding of diabetes in the older person. Diabet Med. 2011;28:117-122.

21. Rosen MI, Beauvais JE, Rigsby MO, Salahi JT, Ryan CE, Cramer JA. Neuropsychological correlates of suboptimal adherence to metformin. J Behav Med. 2003;26:349-360.

22. Trimble LA, Sundberg S, Markham L, Janicijevic S, Beattie L, Meneilly GS. Value of the Clock Drawing Test to predict problems with insulin skills in older adults. Can J Diabetes. 2005;29:102-104.
23. Cavanaugh K, Huizinga MM, Wallston KA, et al. Association of numeracy and diabetes control. Ann Intern Med. 2008;148:737-746.

24. Primozic S, Tavcar R, Avbelj M, Dernovsek MZ, Oblak MR. Specific cognitive abilities are associated with diabetes self-management behaviour among patients with type 2 diabetes. Diabetes Res Clin Pract. 2012;95:48-54.

25. Hamilton M. A rating scale for depression. J Neurol Neurosurg Psychiatry. 1960;23:56-62.

26. Feil D, Zhu C, Sultzer D. The relationship between cognitive impairment and diabetes self-management in a population based community sample of older adults with type 2 diabetes. J Behav Med. 2012;35:190-199.

27. Vedhara K, Wadsworth E, Norman P, et al. Habitual prospective memory in elderly patients with type 2 diabetes: implications for medication adherence. Psychol Health Med. 2004;9:17-27.

28. Moher D, Liberati A, Tetzlaff J, Altman DG; The PRISMA Group. Preferred reporting items for systematic reviews and meta-analyses: the PRISMA statement. Ann Intern Med. 2009;151(4):264-269.
Psychology Research and Behavior Management

\section{Publish your work in this journal}

Psychology Research and Behavior Management is an international, peerreviewed, open access journal focusing on the science of psychology and its application in behavior management to develop improved outcomes in the clinical, educational, sports and business arenas. Specific topics covered include: Neuroscience, memory \& decision making; Behavior

\section{Dovepress}

modification \& management; Clinical applications; Business \& sports performance management; Social and developmental studies; Animal studies. The manuscript management system is completely online and includes a quick and fair peer-review system. Visit http://www.dovepress. com/testimonials.php to read real quotes from published authors. 